\title{
Effect of Refined Surface Domains Walls on the Core Losses Components in GO Silicon Steel at Different Frequencies
}

\author{
I. Petrshynets ${ }^{a, *}$, F. KováC̆ ${ }^{a}$, V. PuchÝ ${ }^{a}$, J. FÜZeR $^{b}$, \\ P. KOLLÁR ${ }^{b}$ AND M. PODOBOVÁ ${ }^{a}$ \\ ${ }^{a}$ Division of Metals Systems, Institute of Materials Research, Watsonova 47, 04001 Košice, Slovakia \\ ${ }^{b}$ Institute of Physics, Faculty of Science, P.J. Safarik University, Park Angelinum 9, 04154 Košice, Slovakia \\ The fiber laser scribing was performed on the surface of grain-oriented (GO) electrical steel, in order to \\ investigate the influence of surface magnetic domains modification on its magnetic losses components. The domains \\ refinement was carried out by the laser beam in continuous regime with the power up to $30 \mathrm{~W}$, and two constant \\ distances between the neighbouring scribing lines, respectively, $4 \mathrm{~mm}$ and $10 \mathrm{~mm}$. It was shown that laser radiation \\ induces the thermal residual stresses at the area of interaction with steel surface that allow to effectively refined \\ surface domain structures, and decrease the hysteresis losses and excess losses of treated steel. The total losses and \\ its components on samples treated by the individual laser scribing regimes were measured in DC and AC magnetic \\ field up to $50 \mathrm{~Hz}$ at maximum flux density of $1.5 \mathrm{~T}$. The obtained results clearly show that experimental samples \\ were characterized by significant decrease of hysteresis losses and excess losses after the surface laser processing.
}

DOI: 10.12693/APhysPolA.137.896

PACS/topics: Laser scribing, Grain-oriented steel, domains refinement

\section{Introduction}

Grain-oriented (GO) electrical steels are used in power distribution networks as transformer core. Its iron loss properties have a large effect on transformer energy efficiency [1]. The magnetic properties of these steels are closely related to both the microstructure and the strong $\{110\}\langle 001\rangle$ texture (also called the Goss-type texture), and importantly they provide very low power loss and high permeability in the rolling direction. On the other hand, GO steel has large grain size which manifests itself in two ways: forming large magnetic domains, and giving rise to a large excess loss when the domain walls move for a longer distance under the action of alternating field [2].

The total magnetic losses $P_{s}$ are used to characterize the quality of electrical steel. According to [3], $P_{s}$ can be separated into three different loss components, namely specific hysteresis $P_{\text {hyst }}$, classical eddy current $P_{\text {clas }}$, and excess or anomalous loss $P_{\text {exc. }}$. All three loss components are related to eddy currents, but the way and their role of generation inside electrical steel is different. Hysteresis loss occurs in the system when small domain wall segments alternate between the local minima of system free energy, creating localized eddy currents. The classical eddy current is created in cross section perpendicular to magnetic field vector. The eddy current loss is determined by the geometry and conductivity of the material. The excess loss is created by eddy currents around domain walls that moved by the driving action of

*corresponding author; e-mail: ipetryshynets@saske.sk the external field. Since excess loss depends on the scale of magnetic domains, their size needs to be decreased. Laser scribing produces localized stresses at the surface of the sheet leading to a domains refinement which significantly reduces excess loss [4]. Using that kind of laser beam source typically one can achieved core loss improvement of about $10 \%$. The theoretically predicted core loss improvement of $70 \%$ is huge motivation for further developments of the refinement process [5].

In our previous work [6], we investigated the influence of the surface laser scribing on the final magnetic properties of electrical steels. It was shown that at the optimal conditions of laser processing it is possible to significantly decrease the final total losses. However, the aims of this work are focused on the investigation of surface thermal residual stresses achieved by laser radiation, and thus the influence on the decrease of the components of total losses of investigated GO silicon steels.

\section{Experimental procedure}

The experimental laser-treated samples were a commercially available Fe-3.2Si grain-oriented steel grade, classified as M130-28A, with $0.28 \mathrm{~mm}$ sheet thickness. The laser scribing was carried out on the steel samples surface prepared by electric spark cutting in form of strips with dimensions of $3 \times 8 \mathrm{~cm}^{2}$. The longest side of samples was parallel to the rolling direction.

The laser scribing process was performed on the surface of experimental samples by means of "TruLaser Station 3003" fiber laser with wavelength 1064 nm working in continuous regime. The refinement of surface domain structures was carried out in air by laser beam with power density in the range from $12 \mathrm{~W}$ up to $30 \mathrm{~W}$. 
When applying scribing process, the laser beam spot on the samples surface with $50 \mu \mathrm{m}$ in size moved with the velocity of $100 \mathrm{~mm} / \mathrm{s}$. The laser scribing was performed in form of lines on the steels surface perpendicularly to the rolling direction. The distance between the lines was selected as $4 \mathrm{~mm}$ and $10 \mathrm{~mm}$, respectively.

The magnetic properties of experimental samples after laser scribing were investigated by means of total losses which were measured in $\mathrm{DC}$ and $\mathrm{AC}$ magnetic field. The $\mathrm{AC} / \mathrm{DC}$ hysteresis loops in frequency range $\mathrm{DC}-50 \mathrm{~Hz}$ were measured by an $\mathrm{AC} / \mathrm{DC}$ Permeameter $\mathrm{AMH}-1 \mathrm{~K}-\mathrm{S}$ at the maximum flux density of $1.5 \mathrm{~T}$.

The most representative samples were selected for the texture analysis which was carried out by an electron back scattered diffraction (EBSD) method. The magnetic domains were observed by means of Bitter method which is based on the use magnetic colloid suspension. The nanoindentation measurements in the area of heat-affected zone cross-section were carried out by the nanohardness machine "Agilent G200"

\section{Result and discussion}

The magnetic domains structure induced by weak external magnetic field of permanent magnet on the surface of investigated samples is imaged in Fig. 1. Here, the Fig. 1a demonstrates the $180^{\circ}$ domain walls of basic domains which is localized inside of neighbouring grains. Note that longitudinal domains of width about the $50 \mu \mathrm{m}$ are oriented along the magnetization direction. The optical micrograph of domain structure in the area of heat affected line obtained by laser beam with power of $24 \mathrm{~W}$, is shown in Fig. 1b. This result clearly presents that laser track divides the longitudinal $180^{\circ}$ magnetic domain.

The microstructural features of heat affected zones at the subsurface region of experimental steels obtained after the laser processing by beam power of $12 \mathrm{~W}, 24 \mathrm{~W}$, and $30 \mathrm{~W}$, are shown in Fig. 2. The SEM micrographs present that heat affected zone is formed by clearly visible dividing line between initial grain structure and structure created under laser affected surface. These results, as well as the visualization of laser track in Fig. 2b, clearly demonstrate that heat affected zone size under the surface depends on the energy density of laser beam. The increase of laser beam power provides the increase subsurface spot size induced by combination of rapidly heating and cooling processes.

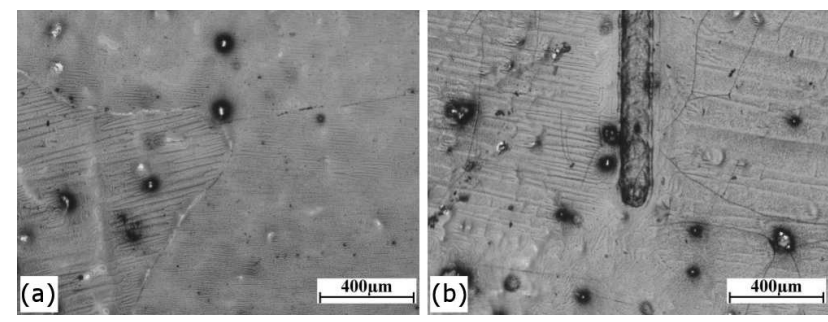

Fig. 1. The surface domains structure of GO steel near (a) the grain boundary, and (b) the laser scribed line.

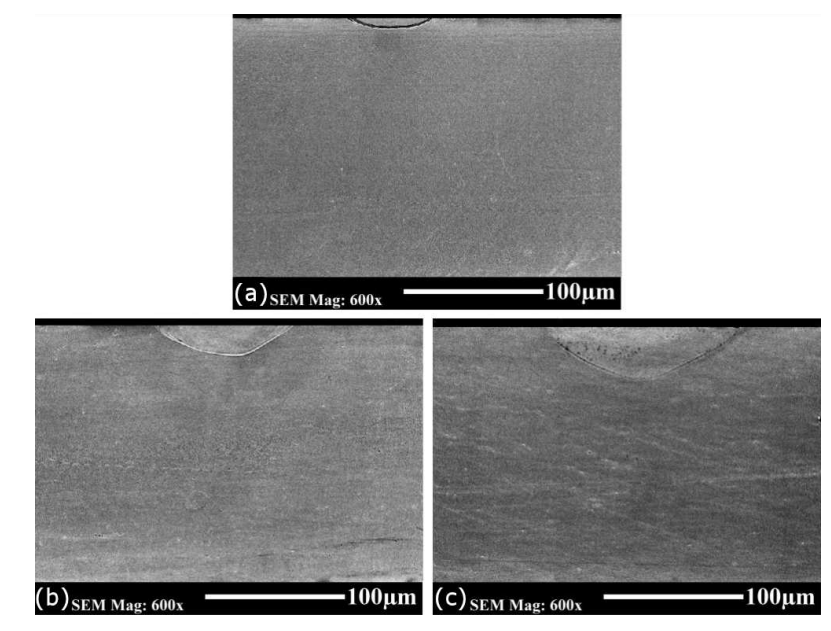

Fig. 2. The microstructure of cross-section of investigated GO steel with heat affected zone obtained after laser treatment with beam power of (a) $12 \mathrm{~W}$, (b) $24 \mathrm{~W}$, and (c) $30 \mathrm{~W}$.

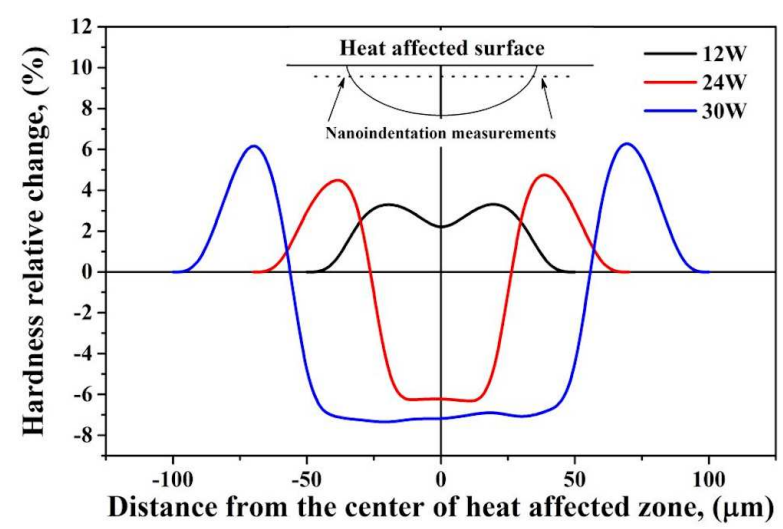

Fig. 3. Dependence of hardness relative change through the laser affected spot on laser beam power.

The investigation of substructural changes in the subsurface area of laser track was carried out by nanoindentation testing, and is presented in Fig. 3. The hardness relative change was measured through the laser induced spot parallel to the surface of treated steel, and schematically shown at the top of Fig. 3. The obtained curves clearly indicate that hardness profile of subsurface heat affected spot characterized by inhomogeneous distribution. It is seen that central part of the inside structure is characterized by decrease of hardness. On the other hand, the increase of hardness was observed near the dividing line in all investigated spots. This effect could be related to the inhomogeneous distribution of laser-induced residual thermal stresses and plastic deformation, which are created after the laser scribing due to the rapidly solidification (hardening).

The state of crystallographic orientation of grains treated by laser radiation with different power was investigated by EBSD (Electron backscatter diffraction) measurements. The most relevant fiber for the grain-oriented 


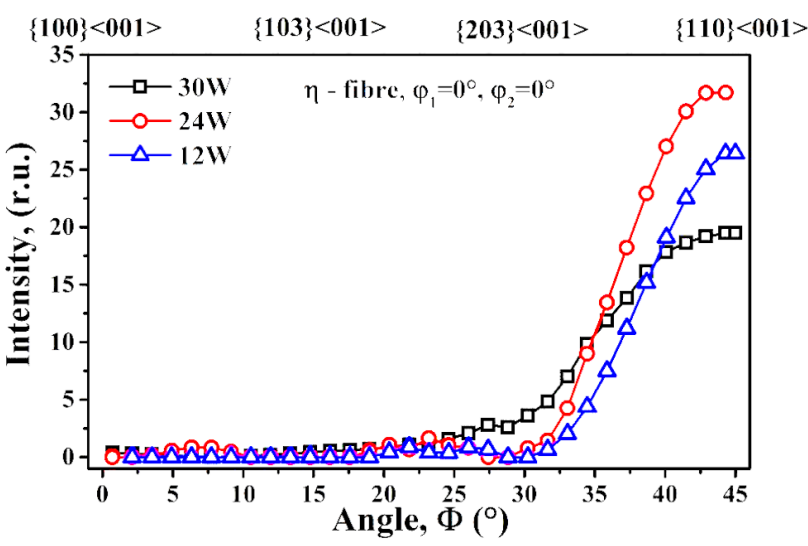

Fig. 4. The dependence of orientation density of laser affected grains along $\eta$-fiber on the power of laser beam.

steel is so called $\eta$-fiber, which presents the intensity of Goss crystallographic texture. The dependence of orientation density along $\eta$-fiber on the power of laser beam is presented in Fig. 4. This result indicates that laser beam with power in the range from $12 \mathrm{~W}$ up to $30 \mathrm{~W}$ interacting with the steel surface induces only partial changes of substructure by thermal stresses in the place of contact. However, the texture of the spot induced under the laser track is similar with texture of initial grain and characterized by high intensity of $\{110\}\langle 001\rangle$ Goss component. It could be conclude that laser processing at lower value of irradiation energy density does not destroy the sharpness of Goss crystallographic orientation in treated steel.

The magnetic properties of the laser treated strips at different conditions were evaluated by means of the total losses measurements in DC and AC magnetic field. The dependence of total losses which are presented by three loss components on the laser radiation power, and distance between the neighbouring scribed tracks is presented in Fig. 5.

These results clearly demonstrate that laser scribing conditions significant influence the total losses of treated GO steels. The measurements were carried out on the strips with $4 \mathrm{~mm}$ and $10 \mathrm{~mm}$ distance between scribing lines generated by laser beam with power of $12 \mathrm{~W}$, $18 \mathrm{~W}, 24 \mathrm{~W}$, and $30 \mathrm{~W}$. Better value of total losses was obtained for samples with lower distance between lines.

The analysis of power loses component clearly demonstrate that most significant changes were measured for hysteresis and execs losses. On the other hand, this distribution shows that classical eddy current loss which is determined by geometry of the strips namely their thickness, is characterized by constant value for all samples. As one can see, the lowest values of hysteresis and excess losses were measured for strips with shortest distance between the scribed lines that are induced only by laser radiation with power $24 \mathrm{~W}$. It could be explained by suitable combination of refined $180^{\circ}$ domains size, and value of residual stresses achieved by heat energy of laser beam. The increase of laser beam power deteriorates

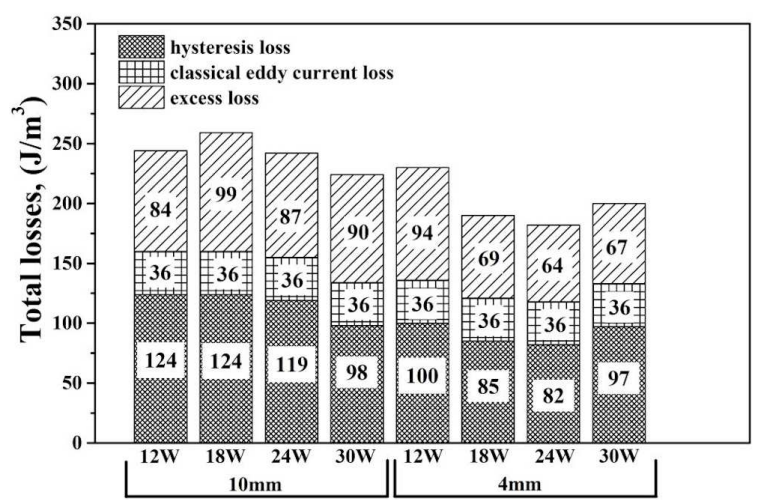

Fig. 5. Total loss and loss components per cycle for laser processed GO steels, where $B_{\max }=1.5 \mathrm{~T}$, $f=50 \mathrm{~Hz}$.

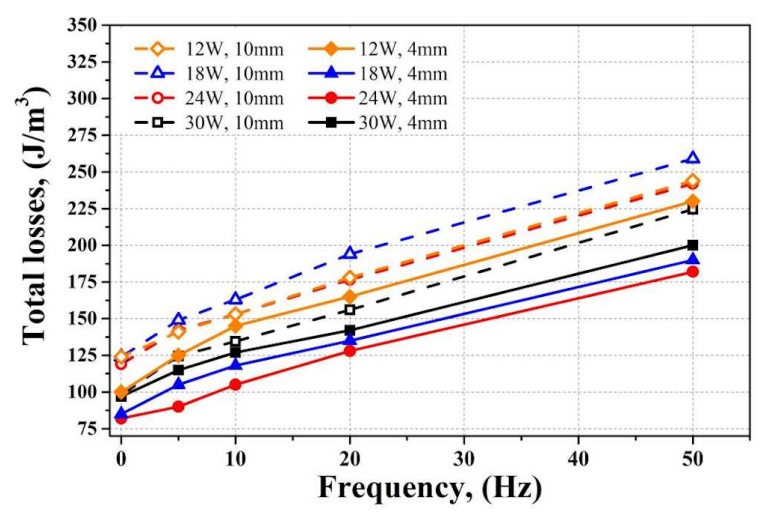

Fig. 6. Total power loss as a function of magnetization frequency, where $B_{\max }=1.5 \mathrm{~T}$, from DC to $50 \mathrm{~Hz}$.

both magnetic components, and may be associated with an increase in thermal residual stresses value, see Fig. 5. The similar tendency of evolution of hysteresis and excess losses was measured for strips with highest distance between laser tracks. In this case, however, the improve of losses components was not so significant as in case of samples with $4 \mathrm{~mm}$ distance between lines.

The dependence of total losses of investigated samples on the frequency of magnetic field is presented in Fig. 6. These measurements indicate that optimal combination of refined domain structures and value of thermal stresses allows to significantly decrease the total losses of GO steel in used frequency range. It could be stated that despite the increase of density of thermal stresses on surface of laser processed strips, that deteriorate its magnetic properties, the appropriate refinement of domains structure in combination with optimal laser power can effectively decrease the resulting total losses.

\section{Conclusions}

The influence of laser scribing on the hysteresis, classical eddy current and excess losses have been investigated. The result clearly shows that the various combination of technical parameters of laser treatment processes affect 
the total power losses in GO electrical steel laminations. The hysteresis loss is significant decreased because of high number of movable $180^{\circ}$ domains walls which were produced by optimal value of thermal stress. The classical eddy current loss is not changed for the treated steel and related to the constant value of lamination thickness and the specific electrical resistivity. This work shows also that excess loss is sensitive to the domain size, and is significantly reduced for the samples with the lowest distance between the scribed lines.

\section{Acknowledgments}

This work was carried out within the projects, which are supported by the Slovak Research and Development Agency under the contract No. APVV-15-0259 and APVV-18-0207. In addition, this work was partially supported by the Slovak Grant Agency VEGA, project No. 2/0066/18 and No. 2/0073/19. This work was supported by the Development Operational Programme Research and Innovation for the project "New unconventional magnetic materials for applications", ITMS: 313011T544, co-funded by the European Regional Development Fund (ERDF).

\section{References}

[1] K. Price, B. Goode, D. Power, Ironmak. Steelmak. 43, 636 (2016).

[2] Z. Xia, Y. Kang, Q. Wang, J. Magn. Magn. Mater. 320, 3229 (2008).

[3] G. Bertotti, IEEE Trans. Magn. 24, 661 (1988).

[4] R. Schäfer, I. Soldatov, S. Arai, J. Magn. Magn. Mater. 474, 221 (2019).

[5] P. Rauscher, B.Betz, J. Hauptmann, A. Wetzig, E. Beyer, C. Grünzweig, Sci. Rep. 6, 38307 (2016).

[6] I. Petryshynets, F. Kováč, V. Puchý, M. Šebek, J. Füzer, P. Kollár, AIP Advances. 8, 047604 (2018). 